\title{
Normdurchsetzung durch Privatrecht
}

\author{
Moritz Brinkmann* \\ Rezension zu Dörte Pölzig, Normdurchsetzung durch Privatrecht, Tübingen (Mohr Siebeck - Heidel- \\ berger Rechtswissenschaftliche Abhandlungen, Band 6) 2012, 652 Seiten, ISBN 978-3-16-151868-3
}

Welche Rückwirkungen ergeben sich für das Privatrecht daraus, dass der Gesetzgeber zunehmend auf privatrechtliche Instrumente zurückgreift, um öffentliche Interessen zu verwirklichen? Dieser Frage widmet sich Dörte Pölzig in ihrer Schrift „Normdurchsetzung durch Privatrecht“, mit der sich die Verfasserin im Jahr 2010 an der Universität Heidelberg habilitierte. Das Phänomen der privaten Rechtsdurchsetzung untersucht Pölzig am Beispiel wirtschaftsrechtlicher Materien, nämlich anhand des Lauterkeits-, des Kartell- und des Kapitalmarktrechts. Dabei berücksichtigt Pölzig jeweils auch die Fragen und Herausforderungen, die sich für das Zivilprozessrecht ergeben. Die Konzentration auf das Wirtschaftsrecht liegt besonders nah, weil dieses Rechtsgebiet funktional auf die Ordnung und Erhaltung des Markts ausgerichtet ist und ihm dadurch eine "erhöhte“ (S.12 - wohl im Vergleich mit dem allgemeinen Privatrecht) Ausrichtung auf das Gemeinwohl eigen ist. Man darf freilich fragen, inwieweit „Wirtschaftsrecht" überhaupt „Zivilrecht“ ist. Immerhin zitiert Engel (JZ 1995, 213) Mestmäcker mit dem Satz: „Wirtschaftsrecht ist die Fortsetzung des Zivilrechts mit anderen Mitteln“. Vor diesem Hintergrund wären die Grenzen der Rechtsdurchsetzung durch Privatrecht möglicherweise noch deutlicher hervorgetreten, wenn auch Materien untersucht worden wären, die keine „erhöhte Gemeinwohlorientierung “ aufweisen, sondern vorrangig dem Schutz von Individualinteressen dienen. So bleibt offen, ob die Indienstnahme des Privatrechts nur in solchen (Privat-) Rechtsbereichen gelingen kann, die wenigstens auch dem Schutz Allgemeininteressen dienen. Eine stärkere Berücksichtigung des UKlaG, das wohl wegen der Fokussierung auf das Wirtschaftsrecht weitgehend ausgeblendet wird, wäre insoweit möglicherweise fruchtbar gewesen.

Die rund 600 Seiten starke Schrift ist in fünf Teile nebst einer Zusammenfassung der Ergebnisse gegliedert. Nach der Einführung in die Grundlagen der privaten Normdurchsetzung, in der die sich insoweit stellenden Herausforderungen sehr deutlich werden, beschreibt Pölzig im zweiten Teil, inwieweit in den von ihr ausgewählten Rechtsmaterien auf privatrechtliche Normdurchsetzungsmechanismen zurückgegriffen wird. Sie beginnt mit einem Blick auf das U.S.-amerikanische Recht, also gleichsam auf das Mutterland des private law enforcement, wobei sie eine „uneingeschränkte und bedingungslose Übernahme des US-amerikanischen private enforcement in das deutsche Recht" nicht als empfehlenswert erachtet. Stattdessen verfolgt

* Prof. Dr. Moritz Brinkmann lehrt deutsches und internationales Zivilprozessrecht sowie Konfliktmanagement an der Universität Bonn. 
sie einen differenzierten Ansatz und plädiert dafür, einzelne Regelungsinstrumente auf ihre Übertragbarkeit zu überprüfen.

Anschließend wendet Pölzig sich dem Lauterkeitsrecht zu und führt vor, dass dessen Verhaltensnormen nicht nur den Schutz der Mitbewerber betreffen, sondern auch Steuerungsinstrumente der Marktregulierung sind. Sie greift hier auf L. Raiser zurück, der die Anspruchsberechtigten Mitbewerber als „Funktionäre der Gesamtrechtsordnung" beschrieben hat.

Auch für das Kartellrecht diagnostiziert Pölzig, dass die Zuerkennung kartellrechtlicher Ansprüche vorrangig zum Schutze des Wettbewerbs als Institution erfolge, wobei sie auf Karsten Schmidt (AcP 206, 169 ff) verweist. Ausführlich widmet die Verfasserin sich in diesem Zusammenhang auch dem Verfahrensrecht und zeigt, dass die Modifikationen des allgemeinen Zivilverfahrensrechts dem Ziel dienen, die privatrechtliche Normdurchsetzung zu stärken. In diesem Zusammenhang setzt sie sich auch mit der Entscheidung BGHZ 190, $145 \mathrm{ff}$ (ORWI) auseinander, in der die passing-on defense anders als im US-amerikanischen Recht zugelassen wurde. Pölzig meint, dass die Zulassung dieses Einwands der Präventionsfunktion der Haftung zuwider liefe. Daher wendet sie sich dagegen, es im Rahmen der Vorteilsanrechnung zu berücksichtigen, dass der Anspruchsberechtigte den überhöhten Preis an seine Abnehmer weitergereicht hat. Wenn man der Steuerungsfunktion des Wirtschaftsrechts im Allgemeinen und insbesondere des $\$ 33$ GWB den Vorrang vor der Kompensationsfunktion einräumt, ist das im Ausgangspunkt konsequent. Allerdings wäre selbst auf dieser Grundlage zu fragen gewesen, ob nicht die Beweisprobleme den passing-on Einwand am Ende doch weitgehend leer laufen lassen, so dass es gar nicht nötig ist, die Steuerungsfunktion des Kartellrechts gegen das allgemeine Schadensrecht auszuspielen.

Auch für den kapitalmarktrechtlichen Schadensersatzanspruch nach $\mathbb{\$} \$ 37 \mathrm{~b}, 37 \mathrm{c}$ WpHG und die Verzinsungspflicht nach $\$ 38$ WpÜG sowie den Rechtsverlust nach $\$ 59$ WpÜG als Sanktion von Verletzungen der Angebots- und Veröffentlichungspflicht nach $\$ 35$ WpÜG kommt Pölzig zu dem Schluss, dass diese Ansprüche der geschädigten Anleger „lediglich Mittel zum Zweck des Marktschutzes“ seien. Recht ausführlich setzt sie sich in diesem Zusammenhang auch mit der prozessualen Durchsetzung dieser Ansprüche, insbesondere mit dem KapMuG auseinander. Sie hält dessen praktische Bedeutung bei der Bewältigung von Streuschäden mit der herrschenden Meinung für gering, und fordert im Anschluss an Gerhard Wagner (ZEuP 2008, $6,16)$ nach dem Vorbild der kartell- bzw. lauterkeitsrechtlichen Regelungen ein Regelungsinstrument, „das die rationale Apathie der einzelnen Beteiligten überwindet und die Normdurchsetzung in die Hände geeigneter Institutionen legt, die einen ausreichenden Klageanreiz haben“" (S. 241). 
Öffentlich-rechtliche und privatrechtliche Normdurchsetzung beschreibt Pölzig als funktionale Äquivalente, wobei die privatrechtliche Normdurchsetzung vor allem Vollzugsdefizite auf Seiten der Aufsichtsbehörden ausgleichen solle, die durch leere Staatskassen und „begrenzte Kapazitäten“ begründet würden (S.253). Allerdings sieht sie die private Rechtsdurchsetzung dennoch wohl nicht als ein aus der Not geborenes second best, sondern meint, dass ein „optimales Ergebnis (...) ein abgestimmtes Zusammenspiel öffentlich-rechtlicher und privatrechtlicher Sanktionen“ voraussetzt.

Im dritten Teil wendet sich Pölzig der „Legitimation“ des Privatrechts als Steuerungsinstrument zu. Die Legitimation überprüft sie aus verfassungs- und unionsrechtlicher Sicht. Dieser Ansatz ist insofern überraschend als das mögliche Legitimationsdefizit sich vor allem daraus ergeben könnte, dass das Privatrecht durch die Betrauung mit der Aufgabe der Rechtsdurchsetzung möglicherweise seine Eigenständigkeit verliert und sich Zielkonflikte mit dem intrinsischen (manche mögen sagen: ursprünglichem) Ziel des Privatrechts ergeben könnten, Rechtsbeziehungen zwischen Einzelnen zu regeln und Konflikte zu befrieden. So vermisst (jedenfalls der Prozessualist) etwa eine Auseinandersetzung mit der Frage, wie sich das private law enforcement zu dem Ziel des Gesetzgebers verhält, die außergerichtliche Konfliktbeilegung und die vergleichsweise Beendigung von Prozessen zu stärken. Jedenfalls auf den ersten Blick scheinen das widersprüchliche Ansätze zu sein. Das mit Händen zu greifende Spannungsverhältnis zur Dispositionsmaxime und Verhandlungsgrundsatz behandelt Pölzig zwar in anderem Zusammenhang, wenngleich auch dort nur sehr knapp (S. 538 f.) Dass man diesen und anderen grundlegenden Bedenken gegen eine Aufladung des Privatrechts mit der Aufgabe der Rechtsdurchsetzung durch verfassungs- und unionsrechtlichen Überlegungen begegnen kann, erscheint (mir) zweifelhaft. Diese Bedenken werden auch nicht beseitigt, wenn Pölzig beschreibt, wie insbesondere das Unionsrecht das Privatrecht durch das „unionsrechtliche Strukturprinzip der funktionalen Subjektivierung “ (S. 323) überformt.

Die Effizienz privater Rechtsdurchsetzung beleuchtet die Verfasserin, wenn sie das Phänomen der privaten Rechtsdurchsetzung aus rechtsökonomischer Sicht untersucht und hierbei insbesondere das Zusammenspiel öffentlichrechtlicher und privatrechtlicher Steuerungsinstrumente behandelt. Dem öffentlichen Recht will sie dabei das Prä einräumen, wenn die Anreize für die private Rechtsdurchsetzung zu gering sind und wenn es für Einzelne aufwändiger ist, an die zur Rechtsdurchsetzung erforderlichen Informationen zu gelangen als für Behörden. Insgesamt sei eine öffentlich-rechtliche Durchsetzung um so eher ökonomisch gerechtfertigt, je höher die drohenden Folgekosten eines rechtswidrigen Handelns seien (S. 392). Diese Aussage erweckt ein wenig den Eindruck, dass die Verfasserin die private Rechtsdurchsetzung als zwar billiges, aber im Vergleich mit dem öffentlichen Recht nicht gleich wirksa- 
mes Instrument ansieht. Ihre Ergebnisse rechtfertigen eine solche Skepsis meines Erachtens nicht.

Der sehr lesenwerte vierte Teil ist den allgemeinen Lehren der privatrechtlichen Normdurchsetzung gewidmet. Pölzig behandelt hier ausführlich die „Rechtsnatur der privatrechtlichen Normdurchsetzung " und kommt zu dem Ergebnis, dass wir es hier mit „einer von dem tradierten Konzept der Privatnützigkeit abweichenden Funktionsweise privatrechtlicher Regelungen “ zu tun haben, nämlich in dem Sinn, dass die Ansprüche, die das Recht begründet, nicht dem Schutz von Individualinteressen dienen, sondern dem Schutz der Institution Markt. Die Kategorie des subjektiven Rechts werde hier funktionalisiert, um objektiv-rechtlichen Institutionenschutz zu gewährleisten. Diese funktionale Deutung zeigt, welche Sprengkraft das Phänomen der privaten Rechtsdurchsetzung für unser Verständnis von Privatrecht besitzt. Den Konsequenzen dieser Umfunktionierung spürt Pölzig ausführlich in materieller und eher knapp in prozessualer und kollisionsrechtlicher (Art. 6 Rom II-VO) Hinsicht nach.

Der fünfte Teil ist der durch die Aufgabe der Rechtsdurchsetzung veränderten Rolle des Privatrechts als Teil der Gesamtrechtsordnung gewidmet. Pölzig schreibt, dass die „Normdurchsetzung im Wirtschaftsrecht (...) beispielhaft dafür [sei], dass die theoretisch klare Trennung zwischen Privatrecht und öffentlichem Recht bei einer funktionalen und problemlösungsorientierten Regelung verschwimmen “ könne.

Der Satz wirft die Frage auf, welche anderen Beispiele es außerhalb des Wirtschaftsrechts für diese „funktionale Äquivalenz" von Privatrecht und öffentlichem Recht gibt. Denkbare „Verdächtige“ sind (ohne jeden Anspruch auf Vollständigkeit) das Verbraucherschutzrecht, das Recht der Produkt-, Lebensmittel- und Arzneisicherheit, das Antidiskriminierungsrecht, das Umweltrecht oder das Baurecht. Insofern steht die private Rechtsdurchsetzung, die im Wirtschaftsrecht wie Pölzigs Schrift zeigt schon längst Praxis ist, insgesamt wohl eher am Anfang ihrer Entwicklung. Über den hiermit einhergehenden Bedeutungszuwachs für das Privatrecht mag sich der Privatrechtler freuen, für die Probleme und Herausforderungen, die diese Entwicklung für Grundpfeiler des Privatrechts haben kann, sollte er zwei offene Augen haben. In welche Richtung(en) der Blick zu gehen hat, führt Pölzig sehr eindrucksvoll vor. 